\title{
A construção de um novo instrumento para avaliar correlatos implícitos dos sintomas do transtorno obsessivo-compulsivo: primeira versão do Teste de Associação Implícita
}

\author{
The construction of a new instrument to assess implicit correlates of obsessive-compulsive disorder symptoms: \\ first version of the Implicit Association Test
}

\author{
Mara Sizino da Victoria ${ }^{1}$, Leonardo F. FonTENELLE ${ }^{1}$ \\ 'Departamento de Psiquiatria e Saúde Mental do Instituto de Psiquiatria da Universidade Federal do Rio de Janeiro (IPUB/UFRJ), Programa de Ansiedade e Depressão
}

Recebido: 10/6/2009 - Aceito: 29/9/2009

\begin{abstract}
Resumo
Contexto: A inexistência de uma medida implícita para sintomas do transtorno obsessivo-compulsivo (TOC) limita a avaliação às escalas e aos inventários tradicionais. Objetivos: $\mathrm{O}$ objetivo foi construir um instrumento de avaliação de sintomas obsessivo-compulsivos que independa da autoavaliação pelo examinando [por exemplo, o Teste de Associação Implícita para transtorno obsessivo-compulsivo (TAI-TOC)]. Métodos: A fim de construir o TAI-TOC, foram consultados (1) estudos anteriores que utilizaram o TAI para avaliação de outros sintomas psiquiátricos, (2) psiquiatras e psicólogos com experiência na avaliação e tratamento de pacientes com TOC e (3) os próprios pacientes com TOC. Resultados: Estímulos verbais e visuais foram selecionados para cada dimensão dos sintomas obsessivo-compulsivos (contaminação e lavagem, obsessões de checagem, simetria e colecionismo). Um software projetado para mensurar o tempo de reação em milissegundos (um programa para associação implícita) foi desenvolvido. Uma versão final do TAI-TOC foi obtida. Conclusões: O TAI-TOC expande o arsenal existente para avaliação dos sintomas obsessivo-compulsivos, especialmente naqueles indivíduos que informam mal sobre seus sintomas.
\end{abstract}

Victoria MS, Fontenelle LF / Rev Psiq Clín. 2010;37(5):189-94

Palavras-chave: Transtorno obsessivo-compulsivo, memória implícita, Teste de Associação Implícita.

\begin{abstract}
Background: The lack of an implicit measure for the obsessive-compulsive disorder symptoms limits its assessment to the traditional scales and inventories. Objectives: The aim is the construction of an instrument for the evaluation of obsessive-compulsive symptoms that is independent from examinee's self-evaluation [i.e. the Implicit Association Test for obsessive-compulsive disorder (IAT-OCD)]. Methods: In order to build the IAT-OCD, we consulted (1) previous studies that employed the IAT for the evaluation of other psychiatric symptoms; (2) expert psychiatrists and psychologists with experience in the assessment and treatment of patients with OCD; and (3) patients with OCD themselves. Results: Specific verbal and visual stimuli were selected for each obsessive-compulsive symptom dimensions (contamination-washing, obsessions-checking, symmetry and hoarding). A software designed to measure reaction time in miliseconds (a proxy for implicit association) was developed. A final version of the IAT-OCD was then obtained. Discussion: The IAT-OCD expands the existing armamentarium to evaluate obsessive-compulsive symptoms, especially among those individuals who report badly about their symptoms.
\end{abstract}

Victoria MS, Fontenelle LF / Rev Psiq Clín. 2010;37(5):189-94

Keywords: Obsessive-compulsive disorder, implicit memory, Implicit Association Test.

\section{Introdução}

A avaliação da gravidade de sintomas obsessivo-compulsivos tradicionalmente é feita por escalas e inventários, instrumentos de autoavaliação que incluem aspectos mais comuns dos sintomas. Dentre os instrumentos para adultos, destacam-se: a Yale-Brown Obsessive Compulsive Scale (Y-BOCS), o Obsessive-Compulsive Inventory, o Leyton Obsessional Inventory, o Maudsley Obsessional-Compulsive Inventory (MOCI), a Comprehensive Psychopathological Rating Scale (CRPS), a National Mental Health Obsessive Compulsive Scale e o Padua Inventory (PI)1-5. Entretanto, essas medidas sofrem de limitações naturais impostas pela consciência. As objeções à autoavaliação estão relacionadas com o fato de o indivíduo ter dificuldade de avaliar de forma apropriada o seu verdadeiro estado psicopatológico ${ }^{6,7}$.

Nos últimos anos, uma alternativa interessante começou a surgir mediante testes que medem a memória implícita por meio do tempo de reação à associação de itens. São testes de medida indireta denominados Testes de Associação Implícita (TAI) e foram estudados por um número considerável de pesquisadores ${ }^{8-11}$. O TAI, introduzido por Greenwald et al. em 1998', é uma tarefa simples, baseada na existência de uma associação de dois conceitos por semelhança. Nesse teste, a ideia foi apresentar nomes de flores (por exemplo, tulipa), nomes de insetos (por exemplo, aranha), palavras positivas (por exemplo, amor) e palavras negativas (por exemplo, feio) num computador.
No experimento 1, os nomes das flores eram apresentados junto das palavras positivas e os nomes dos insetos, junto das palavras negativas. No experimento 2, os nomes das flores eram apresentados junto das palavras negativas e os nomes dos insetos, junto das palavras positivas. Os participantes eram instruídos a associar as palavras pressionando uma tecla específica do teclado do computador. Como se pode deduzir, os resultados confirmaram o que os pesquisadores supunham: o tempo de resposta para o experimento 1 foi significativamente menor que para o experimento 2 . A justificativa para tal resultado é que os conceitos de flores e palavras positivas estão mais fortemente associados na memória implícita, pois são utilizados com mais frequência que flores e palavras negativas.

Recentemente, o TAI foi desenvolvido como instrumento para vários sintomas e/ou transtornos psiquiátricos, como ansiedade social $^{12,13}$, fobia a animais ${ }^{14}$, transtorno de personalidade borderline ${ }^{15}$, pânico ${ }^{16}$ e depressão ${ }^{17}$. A lógica dos TAIs parte do tempo de reação, em que se pode deduzir que, quando se pede que um indivíduo associe dois itens previamente determinados, haverá um tempo desde a apresentação desse item até a sua resposta. Quanto menor o tempo de reação a esses itens, mais fortemente eles estariam associados. $\mathrm{Na}$ execução do TAI, o indivíduo é orientado a responder o mais rápido possível, pois a atividade que está envolvida na associação de itens deve ser uma atividade que fuja a qualquer processo de julgamento crítico $^{6}$. 
De fato, a ausência de uma medida implícita válida para sintomas do transtorno obsessivo-compulsivo limita sua avaliação às escalas e aos inventários tradicionais. Com as avaliações clínicas atuais, baseadas em dados explícitos, algumas informações importantes podem não ser detectadas. Isso porque, frequentemente, os pacientes com TOC, ou mesmo indivíduos com sintomas obsessivo-compulsivos subclínicos, descrevem seu estado clínico de forma pouco confiável, seja por prejuízo do insight ou mesmo por constrangimento ou vergonha diante do examinador ${ }^{18}$.

Com efeito, outras medidas implícitas já foram empregadas na avaliação do TOC, sem sucesso. Por exemplo, o perfil de respostas no teste de Stroop emocional, instrumento que mede "atenção seletiva", foi capaz de diferenciar pacientes com TOC de indivíduos saudáveis em apenas uma parcela dos estudos ${ }^{19,20}$, mas não na maioria deles ${ }^{21-24}$. O fato de o teste de Stroop emocional avaliar tempo de reação a estímulos verbais relacionados ao TOC coloca os resultados negativos em cheque. Um paradigma verbal pode não ser suficientemente sensível para detectar um "viés atentivo" ou qualquer outro correlato implícito quando se lida com sintomas que são tão frequentemente agravados por estímulos ou imagens visuais ${ }^{25}$. Infelizmente, a estrutura do teste de Stroop não permite a utilização adequada de estímulos visuais. Essa limitação está superada no TAI, que pode ser modificado a fim de incluir figuras que agravam, ao menos pontualmente, os sintomas obsessivo-compulsivos de pacientes com TOC.

O objetivo do presente trabalho é apresentar uma primeira versão de um teste para identificação de correlatos implícitos dos sintomas obsessivo-compulsivos, o TAI-TOC. Deve-se ressaltar que o TAITOC não pretende substituir as avaliações clínicas tradicionais e os instrumentos disponíveis; a nossa proposta é que seu uso seja apenas complementar, especialmente em ambientes experimentais, porque permite o acesso a correlatos dos sintomas obsessivo-compulsivos que seriam, de outra forma, imperceptíveis.

Com a construção desse instrumento e posterior avaliação psicométrica, se aposta: (1) é possível avaliar manifestações de memória implícita dos sintomas do TOC por meio do TAI; (2) o TAI-TOC pode revelar uma estratégia de processamento de informação diferente entre pacientes e controles; e (3) no futuro o TAI-TOC pode servir como um instrumento auxiliar nas avaliações clínicas em pacientes com TOC, especialmente aqueles com dificuldades para descrever seus sintomas.

\section{Métodos}

A construção do instrumento foi baseado em experiências de pesquisadores anteriores utilizando o TAI para ansiedade 26,27 . A construção do TAI-TOC seguiu as seguintes etapas 28 : escolha dos itens, escolha da modalidade de apresentação dos itens, elaboração das instruções, revisão da primeira versão por especialistas da área e estudo piloto com a amostra para a qual foi construído e seleção dos itens. A escolha dos itens foi feita a partir das dimensões mais comuns do TOC 29,30 , a saber: (1) contaminação e lavagem, (2) simetria, ordenação e arranjo, (3) colecionismo, (4) "obsessões" (i.e., de agressão, sexuais e religiosas) e checagem. Foram escolhidas seis palavras que representam os itens e que resumem os pensamentos obsessivos e rituais compulsivos correspondentes a cada dimensão. A seleção dos itens foi feita a partir da experiência clínica com pacientes com o transtorno. As 12 palavras que representam as obsessões e compulsões encontram-se na tabela 1.

Tabela 1. Itens de obsessão e compulsão que compõem o TAl-TOC

\begin{tabular}{c|c|c}
\hline Dimensão & Itens Obsessão & Itens Compulsão \\
\hline Agressão & Agredir & Checar \\
\hline Sexual & Estuprar & Conferir \\
\hline Religião & Pecar & Rezar \\
\hline Contaminação & Sujar & Limpar \\
\hline Simetria & Bagunçar & Organizar \\
\hline Colecionismo & Perder & Guardar \\
\hline
\end{tabular}

Os itens de obsessões e compulsões fazem parte da classe de itens denominada de "nervoso" versus "calmo", respectivamente. Conforme organização estrutural do teste, buscou-se eleger outras duas categorias de palavras para as associações. Esta classe foi denominada "bom" versus "ruim". A seleção dos itens foi feita pela semântica das palavras (Tabela 2).

Tabela 2. Itens de bom e ruim que compõem o TAI-TOC

\begin{tabular}{l|l}
\hline Itens da categoria Bom & Itens da categoria Ruim \\
\hline Sereno & Ansioso \\
\hline Positivo & Negativo \\
\hline Aliviado & Preocupado \\
\hline Tranquilo & Tenso \\
\hline Seguro & Apreensivo \\
\hline Relaxado & Temeroso \\
\hline
\end{tabular}

A apresentação dos itens seguiu o modelo de TAIs anteriores ${ }^{9,10}$ Os itens aparecem na tela do monitor aleatoriamente, respeitando o número de repetições por palavra.

As instruções do instrumento seguiram o padrão estabelecido por outros TẢs e estão disponíveis no site do projeto implícito: https://implicit.harvard.edu/implicit/31.

Após a escolha dos itens, uma versão do teste foi submetida a quatro especialistas, dois psicólogos e dois psiquiatras com experiência de mais de três anos na avaliação e tratamento do TOC, do Instituto de Psiquiatria da Universidade Federal do Rio de Janeiro (IPUB/UFRJ), para julgamento. Os itens foram avaliados segundo a frequência de sintomas para as categorias "calmo" versus "nervoso" e semântica das palavras para as categorias "bom" versus "ruim".

A inclusão de estímulos visuais foi sugerida para acompanhar os itens de obsessões e compulsões. A utilização de imagens já é feita em outros TAIs, como em https://implicit.harvard.edu/implicit/31. Para a seleção de imagens, foi feito um levantamento bibliográfico de instrumentos que utilizam imagens como recurso. Desse levantamento e seleção, dois instrumentos foram considerados condizentes com o objetivo: o International Affective Picture System (IAPS) ${ }^{32}$, adaptado às normas brasileiras ${ }^{33}$, e o Maudsley Obsessive Compulsive Stimuli Set (MOCCS) ${ }^{34}$. Três imagens foram selecionadas desses instrumentos (2 do IAPS e 1 do MOCCS); quatro imagens foram selecionadas do Google imagens; cinco imagens foram produzidas pelos pesquisadores.

Após o julgamento por especialistas, cinco pacientes foram selecionados aleatoriamente dentre aqueles que já estão em tratamento no Programa de Ansiedade e Depressão do Instituto de Psiquiatria da UFRJ (IPUB/UFRJ). O objetivo da participação dos pacientes foi testar a aceitabilidade dos itens selecionados. Uma versão foi produzida por meio do software Java para ser submetida aos pacientes. A amostra clínica avaliou a coerência dos itens às categorias, a semântica das palavras (significado) e equivalência das palavras com as imagens. A modificação das palavras após a avaliação dos pacientes encontra-se na tabela 3 .

Tabela 3. Itens finais que compõem o TAI-TOC

\begin{tabular}{c|c|c|c}
\hline $\begin{array}{c}\text { Categoria } \\
\text { "nervoso" }\end{array}$ & $\begin{array}{c}\text { Categoria } \\
\text { "calmo" }\end{array}$ & $\begin{array}{c}\text { Categoria } \\
\text { "bom" }\end{array}$ & $\begin{array}{c}\text { Categoria } \\
\text { "ruim" }\end{array}$ \\
\hline Agressão & Verificado & Sereno & Ansioso \\
\hline Estupro & Conferido & Positivo & Negativo \\
\hline Pecado & Perdoado & Aliviado & Preocupado \\
\hline Sujo & Limpo & Tranquilo & Tenso \\
\hline Bagunça & Organizado & Seguro & Inseguro \\
\hline Perda & Guardado & Bom & Ruim \\
\hline
\end{tabular}

As palavras da categoria "calmo" foram modificadas de verbos no infinitivo impessoal para o particípio. Portanto, "conferir" foi alterado para "conferido", "limpar" para "limpo", "organizar" para "organizado", "guardar" para "guardado". Os itens "checar" e "rezar" foram transformados em "verificado" e "perdoado" por causa da 
imagem apresentada, por sugestão da amostra clínica. A mudança se justifica porque a nomeação no particípio se associa mais diretamente a "calmo" que no infinitivo. Exemplo: "limpar" é diferente de "limpo", o que propicia uma associação mais direta à categoria "calmo".

A categoria "nervoso" sofreu variação do infinitivo para substantivos e adjetivos. Nesse sentido, "agredir" foi modificado para "agressão", "estuprar" para "estupro", "pecar" para "pecado", "sujar" para "sujo", "bagunçar" para "bagunça”, "perder" para "perda”.

Nas categorias "bom" e "ruim", algumas palavras foram trocadas: "apreensivo" para "inseguro", "relaxado" para "bom" e "temeroso" para "ruim". As palavras foram julgadas como incompreensíveis e, por isso, seguiram a sugestão dos pacientes.

Após a participação da amostra clínica, foi possível construir a primeira versão do TAI-TOC, descrita a seguir.

\section{Resultados}

O TAI-TOC é um instrumento de tarefa cronometrada que é respondido no computador. A apresentação da tela é em fundo preto e as palavras aparecem em branco e amarelo. Um exemplo de apresentação de cada bloco encontra-se na figura 1 .

A tarefa consiste no aparecimento de palavras, uma palavra ou um par de palavras à esquerda da tela do monitor; uma palavra ou um par de palavras à direita da tela do monitor e uma palavra junto com uma imagem ao centro da tela do monitor. A palavra e a imagem que aparecem no centro do monitor são aquelas em que o participante deve avaliar se estão associadas à palavra ou par de palavras da direita ou da esquerda. Caso seja com a da esquerda, ele deve apertar a tecla "e" do teclado do computador; caso seja com a da direita, ele deve apertar a tecla "i". Se a resposta do participante for errada, um "X" vermelho aparecerá na tela e ele, então, deve dar a resposta correta. São consideradas as associações corretas a combinação de todos os itens que compõem a categoria "nervoso" com "ruim" e "calmo" com "bom".

Após o início do teste, as palavras aparecem numa frequência equilibrada. O teste foi estruturado em sete blocos, seguindo a organização estrutural de Egloff et al. ${ }^{26,27}$. Os blocos 1 e 2, compostos por 24 tentativas, e o bloco 5, composto por 48 tentativas, são relacionados com tarefas de associação simples (uma palavra à esquerda, uma à direita e uma no centro junto com uma imagem). Os blocos 3 e 4 (bloco 3, composto por 24 tentativas, e bloco 4, por 48) e 6 e 7 (bloco 6, composto por 24 tentativas, e bloco 7 , por 48) são relacionados com tarefas de associação dupla (um par de palavras à esquerda, um par à direita e uma palavra no meio junto com uma imagem). No total, são 240 tentativas, a partir das quais resultarão 240 tempos de reação. O esquema do teste está apresentado na tabela 4

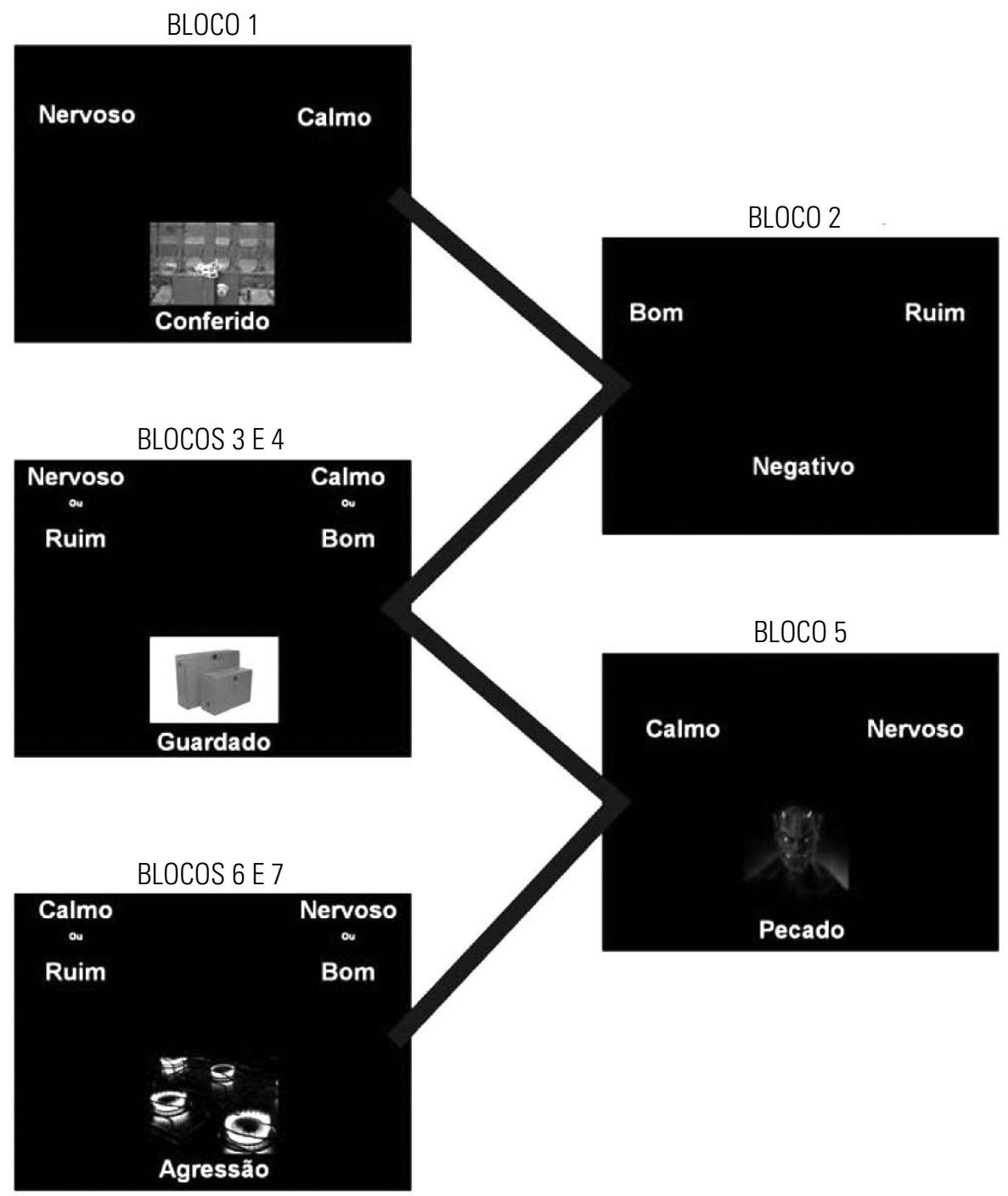

Figura 1. Exemplo de uma tela do TAI-TOC. 
Tabela 4. Organização do TAl-TOC por bloco

\begin{tabular}{|c|c|c|c|c|c|c|c|}
\hline & BLOCO 1 & BLOCO 2 & BLOCO 3 & BLOCO 4 & BLOCO 5 & BLOCO 6 & BLOCO 7 \\
\hline DESCRIÇÃO & $\begin{array}{l}\text { Associação } \\
\text { simples }\end{array}$ & $\begin{array}{c}\text { Associação } \\
\text { simples }\end{array}$ & $\begin{array}{l}\text { Associação dupla } \\
\text { entre os conceitos } \\
\text { do bloco } 1 \text { e } \\
\text { bloco } 2\end{array}$ & $\begin{array}{l}\text { Associação dupla } \\
\text { entre os conceitos } \\
\text { do bloco } 1 \text { e } \\
\text { bloco } 2\end{array}$ & $\begin{array}{c}\text { Associação simples } \\
\text { (invertido do } \\
\text { bloco 1) }\end{array}$ & $\begin{array}{l}\text { Associação dupla } \\
\text { entre os conceitos } \\
\text { do bloco } 1 \text { e } \\
\text { bloco } 2 \\
\text { (invertido do bloco } \\
3 \text { e 4) }\end{array}$ & $\begin{array}{l}\text { Associação dupla } \\
\text { entre os conceitos } \\
\text { do bloco } 1 \text { e } \\
\text { bloco } 2 \\
\text { (invertido do bloco } \\
3 \text { e 4) }\end{array}$ \\
\hline $\begin{array}{l}\text { CATEGORIAS } \\
\text { (palavras da } \\
\text { esquerda e da } \\
\text { direita) }\end{array}$ & $\begin{array}{l}\text { Nervoso } \\
\text { Calmo }\end{array}$ & $\begin{array}{l}\text { Bom } \\
\text { Ruim }\end{array}$ & $\begin{array}{l}\text { Nervoso ou Ruim } \\
\text { Calmo ou Bom }\end{array}$ & $\begin{array}{l}\text { Nervoso ou Ruim } \\
\text { Calmo ou Bom }\end{array}$ & $\begin{array}{l}\text { Calmo } \\
\text { Nervoso }\end{array}$ & $\begin{array}{l}\text { Calmo ou Ruim } \\
\text { Nervoso ou Bom }\end{array}$ & $\begin{array}{l}\text { Calmo ou Ruim } \\
\text { Nervoso ou Bom }\end{array}$ \\
\hline $\begin{array}{l}\text { EXEMPLO } \\
\text { (palavras do centro) }\end{array}$ & $\begin{array}{c}\text { Agressão } \\
\text { Conferido } \\
\text { Sujo } \\
\text { Limpo }\end{array}$ & $\begin{array}{l}\text { Sereno } \\
\text { Ansioso } \\
\text { Seguro } \\
\text { Inseguro }\end{array}$ & $\begin{array}{l}\text { Agressão } \\
\text { Sereno } \\
\text { Conferido } \\
\text { Ansioso }\end{array}$ & $\begin{array}{l}\text { Agressão } \\
\text { Sereno } \\
\text { Conferido } \\
\text { Ansioso }\end{array}$ & $\begin{array}{l}\text { Agressão } \\
\text { Conferido } \\
\text { Sujo } \\
\text { Limpo }\end{array}$ & $\begin{array}{l}\text { Agressão } \\
\text { Sereno } \\
\text { Conferido } \\
\text { Ansioso }\end{array}$ & $\begin{array}{l}\text { Agressão } \\
\text { Sereno } \\
\text { Conferido } \\
\text { Ansioso }\end{array}$ \\
\hline $\mathrm{N}^{\circ}$ DE ITENS & 24 & 24 & 24 & 48 & 48 & 24 & 48 \\
\hline
\end{tabular}

No bloco 1, a tarefa de associação é simples e relacionada com o conceito de "nervoso X calmo". O bloco 2 também é de associação simples e está relacionado com o conceito de "bom X ruim". Os blocos de interesse e de dupla associação são os 3 e 4 e o 6 e 7. Os blocos 3 e 4 são de associação dos conceitos "nervoso ou ruim" X "calmo ou bom”. Esses blocos são idênticos; o bloco 3 é de treino. O bloco 5 tem exatamente a mesma associação do bloco 1, mas de forma invertida. Os blocos 6 e 7 são idênticos ao 3 e 4, só que invertidos: "calmo ou ruim" X "nervoso ou bom". Cada bloco de interesse (3 e 4, 6 e 7) é composto por 72 tentativas, juntos, e as primeiras 24 respostas são consideradas de treino.

As descrições dos estímulos visuais da categoria "nervoso" são as seguintes:

- Agressão: fogão com as quatro bocas acesas.

- Estupro: homem com uma faca no pescoço de uma mulher.

- Pecado: rosto de diabo.

- Sujo: vaso sanitário com fezes.

- Bagunça: sapatos soltos no chão.

- Perda: contas e extratos bancários desordenados.

As descrições dos estímulos visuais da categoria "calmo" são as seguintes:

- Verificado: fogão com as quatro bocas apagadas.

- Conferido: portão, corrente e cadeado fechados.

- Perdoado: rosto de Jesus Cristo.

- Limpo: vaso sanitário sem fezes.

- Organizado: sapatos simétricos no chão.

- Guardado: duas caixas-arquivo.

A análise dos resultados é feita pelo tempo de reação de cada item dos blocos 3 e 4 e 6 e 7, registrada em milissegundos. O software gera um arquivo de Excel com todos os tempos de resposta. A execução desse teste dura em torno de 15 minutos.

\section{Discussão}

No presente trabalho, procurou-se apresentar um novo tipo de avaliação para o TOC. O TAI é o tipo de teste mais conhecido para medida implícita. Do ano de 1998 até hoje muitos estudos foram desenvolvidos para avaliar a memória implícita em amostras clínicas ou não ${ }^{6}$. Outras medidas automáticas, como o teste de Stroop, foram estudadas para o TOC ${ }^{21-24}$, demonstrando resultados heterogêneos em termos de tempo de reação perante estímulos verbais. O TAI-TOC é um diferencial no campo das medidas automáticas, porque revela os correlatos mnêmicos implícitos dos sintomas do TOC diante de estímulos visuais, até então não investigadas pelo Stroop, que tem investigado apenas o aspecto atencional e verbal do processamento de informações.

É de longa data o interesse pelo grau de insight ou capacidade de julgamento crítico dos pacientes com TOC em relação aos seus sintomas. O DSM-IV especifica o TOC com insight pobre, quando, na maior parte do tempo durante o episódio atual, o indivíduo não reconhece que as obsessões ou compulsões são excessivas e irracionais. $\mathrm{O}$ TOC com insight pobre tem sido associado à idade de início mais precoce, maior tempo de doença, maiores gravidades de sintomas e extensão do comprometimento funcional e familiar e pior magnitude de resposta ao tratamento ${ }^{35}$. Em um estudo com 78 pacientes diagnosticados pelos critérios do DSM-IV e tratados com inibidores de recaptação de serotonina (ISRSs), 36\% não reconheciam suas obsessões e compulsões como excessivas ${ }^{36}$.

Ainda que a maioria dos pacientes com TOC tenha crítica em relação aos seus sintomas, é sabido também que os pacientes com TOC habitualmente tendem a "esconder" sua condição clínica por causa de vergonha e constrangimento em relação aos sintomas. Por exemplo, pacientes com TOC demoram até 17 anos para procurar e obter atendimento adequado. Muitos pacientes ocultam seus sintomas de familiares próximos, porque pensam que, ao falar de seus medos, eles de fato se realizem. Outros pacientes, especialmente aqueles com obsessões sexuais ou agressivas, temem ser considerados loucos ou perigosos e hospitalizados ou mesmo presos. Não é surpreendente, portanto, que muitos desses pacientes omitam muitos sintomas e sua real gravidade durante uma avaliação psiquiátrica ou psicológica ${ }^{37}$. Isso implica diretamente o comprometimento da qualidade de vida, porque, não buscando tratamento, os sintomas passam a interferir de forma negativa na vida afetiva, social, estudantil ou profissional ${ }^{38,39}$.

A avaliação de associações implícitas subjacentes a sintomas obsessivo-compulsivos tem como objetivo trazer novas perspectivas para o entendimento do processamento de informações no TOC. Em primeiro lugar, um instrumento de medida implícita supera a autoavaliação deficitária dos sintomas obsessivo-compulsivos, porque os pacientes participam de uma avaliação indireta, realizando uma tarefa de associação que pretende atingir o sintoma que passa despercebido pelo paciente ${ }^{6}$. Além disso, o uso de outros TAIs para diversos outros construtos psiquiátricos ${ }^{12-17}$ sugere a sua utilidade para o TOC.

Em segundo lugar, o TAI-TOC pode revelar características similares de funcionamento da memória implícita em pacientes com TOC e em pessoas em risco de desenvolver TOC (por exemplo, familiares de primeiro grau de pacientes com TOC). Isso significa supor que a memória implícita pode mostrar-se como um endofenótipo para o TOC, um traço intermediário entre o nível de sintoma e sua base genética ${ }^{40}$. A identificação de um endofenótipo é necessária e útil, visto que proporciona maior objetividade na seleção de "genes candidatos" em diferentes transtornos mentais. Ampliando, o TAI pode ser um preditor de pessoas em risco de desenvolver TOC, visto que, por semelhança às respostas dos pacientes, pode antecipar um diagnóstico tardio. Espera-se que 
pacientes com TOC tenham latência maior no resultado geral do teste que os controles e que pacientes com TOC apresentem latências diferenciadas para as dimensões de seus sintomas. Além disso, espera-se encontrar um traço comum, como a latência de resposta comum para as mesmas dimensões entre pacientes e seus familiares de primeiro grau.

Por último, a participação da amostra clínica na avaliação da coerência dos itens às categorias, da semântica das palavras e equivalência das palavras com as imagens e na execução da tarefa revelou a viabilidade de resposta por meio dessa nova forma de medida.

Este estudo encontra-se numa fase inicial e, portanto, apresenta algumas limitações: (1) o TAI-TOC ainda não foi testado numa amostra significativa de pacientes que permitisse uma avaliação detalhada das respostas a todos os seus itens; (2) como a faixa de insight em pacientes com TOC é ampla, essas correlações podem ser comprometidas pelas flutuações de insight, assim como pelas oscilações de atenção; e (3) embora fatores ambientais, como aspectos culturais, religiosos e sociais, não sejam contemplados pelo TAI-TOC, devem ser levados em conta em estudos futuros por apresentarem potencial influência na avaliação implícita de seus sintomas. Em um estudo futuro, esperamos avaliar as propriedades psicométricas, por exemplo, fidedignidade e validade, do TAI-TOC a partir de dados coletados numa amostra clínica e sujeitos saudáveis. Esperamos ainda testar a hipótese de que o perfil de respostas no TAI-TOC seja semelhante em pacientes e familiares de primeiro grau, apontando para uma existência de um endofenótipo.

\section{Referências}

1. Araújo LA. Escalas de avaliação do transtorno obsessivo compulsivo em adultos. In: Gorenstein C, Andrade L, Zuardi A, organizadores. Escalas de avaliação clínica em psiquiatria e psicofarmacologia. São Paulo: Lemos; 2000, p. 165-6.

2. Foa EB, Huppert JD, Leiberg S, Langner R, Kichic R, Hajcak G, et al. The obsessive-compulsive inventory: development and validation of a short version. Psychol Assess. 2002;14:485-96.

3. Foa EB, Kozak MJ, Salkovskis PM, Coles ME, Amir N. The validation of a new obsessive compulsive disorder scale: The Obsessive Compulsive Inventory (OCI) Psychol Assess. 1998;10:206-14.

4. Goodman WK, Price LH, Rasmussen SA. The Yale Brown Obsessive Compulsive Scale YBOCS Part I Development, Use and Reliability. Arch Gen Psychiatry. 1989;46:1006-11.

5. Goodman WK, Price LH, Rasmussen AS. The Yale Brown Obsessive Compulsive Scale YBOCS Part II Validity. Arch Gen Psychiatry. 1989;46:1012-6

6. De Houwer J. The Implicit Association Test as a tool for studying dysfunctional associations in psychopathology: strengths and limitations. J Behav Ther Exp Psychiatry. 2002;33:115-33.

7. Fazio RH, Olson MA. Implicit measures in social cognition research: their meaning and use. Annu Rev Psychol. 2003;54:297-327.

8. Greenwald AG, McGhee DE, Schwartz J. Measuring individual differences in implicit cognition: The Implicit Association Test. J Pers Soc Psychol. 1998;74:1464-80.

9. Cunningham WA, Preacher KJ, Banaji MR. Implicit attitude measures: consistency, stability, and convergent vality. Psychol Sci. 2001;12:163-70.

10. Greenwald AG, Rudman LA, Nosek BA, Banaji MR, Farnham SD, Mellott DS. A unified theory of implicit attitudes, stereotypes, self-esteem, and self-concept. Psychol Rev. 2002;109:3-25.

11. Gawronski B. What does the Implicit Association Test measure? A test of the convergent and discriminant validity of prejudice-related IATs. Exp Psychol. 2002;49:171-80.

12. Jong P. Implicit self-esteem and social anxiety: differential selffavouring effects in high and low anxious individuals. Behav Res Ther. 2002;40:501-8.

13. Tanner RJ, Stopa L, De Houwer J. Implicit views of the self in social anxiety. Behav Res Ther. 2006;44:1397-409.

14. Teachman BA, Gregg AP, Wood SR. Implicit associations of fear-relevant stimuli among individuals with snake and spider fears. J Abnorm Psychol. 2001;110:226-35.
15. Rüsch N, Lieb K, Göttler I, Hermann C, Schramm E, Richter H, et al. Shame and implicit self-concept in women with borderline personality disorder. Am J Psychiatry. 2007;164:500-8.

16. Teachman BA, Smith-Janik SB, Saporito J. Information processing biases and panic disorder: relationsships among cognitive and symptom measures. Behav Res Ther. 2007;45:1791-811.

17. Meites TM, Deveney CM, Steele KT, Holmes AJ, Pizzagalli DA. Implicit depression and hopelessness in remitted depressed individuals. Behav Res Ther. 2008;46:1078-84.

18. Matsunaga H, Kiriike N, Matsui T, Oya K, Iwasaki Y, Koshimune K, et al. Obsessive-compulsive disorder with poor insight. Compr Psychiatry. 2002;43:150-7.

19. Lavy E, Van Oppen P, Van den Hout M. Selective processing of emotional information in obsessive compulsive disorder. Behav Res Ther. 1994;32:243-6.

20. Novara C, Sanavio E. Compulsive cheking and selective processing of threatening information. Psychol Rep. 2001;88:1171-81.

21. Unoki K, Kasuga T, Matsushima E, Ohta K. Attentional processing of emotional information in obsessive-compulsive disorder. Psychiatry Clin Neurosci. 1999;53:635-42.

22. Kampman M, Keijsers GP, Verbraak MJ, Naring G, Hoogduin CA. The emotional Stroop: a comparison of panic disorder patients, obsessivecompulsive patients, and normal controls, in two experiments. J Anxiety Disord. 2002;16:425-41.

23. Moritz S, Jacobsen D, Kloss M, Fricke S, Rufer M, Hand I. Examination of emotional Stroop interference in obsessive-compulsive disorder. Behav Res Ther. 2004;42:671-82.

24. Moritz S, Fischer BK, Hottenrott B, Kellner M, Fricke S, Randjbar S, et al. Words may not be enough! No increased emotional Stroop effect in obsessive-compulsive disorder. Behav Res Ther. 2008;46:1101-4.

25. Rachman S. Unwanted intrusive images in obsessive compulsive disorders. J Behav Ther Exp Psychiatry. 2007;38:402-10.

26. Egloff B, Schmukle SC. Predictive validity of an Implicit Association Test for assessing anxiety. J Pers Soc Psychol. 2002;83:1441-55.

27. Egloff B, Schwerdtfeger A, Schmukle SC. Temporal stability of the Implicit Association Teste-anxiety. J Pers Assess. 2005;84:82-8.

28. Adánez GP. Procedimientos de construcción y análisis de tests psicométricos. In: Wechsler SM, Guzzo RSL, organizadores. Avaliação psicológica: perspectiva internacional. São Paulo: Casa do Psicólogo; 1999, p. $57-100$.

29. Mataix-Cols D, Rosario-Campos MC, Leckman JF. A multidimensional model of obsessive-compulsive disorder. Am J Psychiatry. 2005;162: 228-38.

30. Bloch MH, Landeros-Weisenberger A, Rosario MC, Pittenger C, Leckman JF. Meta-analysis of the symptom structure of obsessive-compulsive disorder. Am J Psychiatry. 2008;165:1532-42.

31. Project Implicit - [acessado em 1 mai 2009]. Disponível em: https:// implicit.harvard.edu/implicit/.

32. Lang PJ, Bradley MM, Cuthbert BN. International Affective Picture System (IAPS): Instruction Manual and Affective Rating. Technical Report A-5, The Center for Research in Psychophysiology, University of Florida; 2001.

33. Ribeiro RL, Pompéia S, Bueno OF. Normas brasileiras para o International Affective Picture System (IAPS): comunicação breve. Rev Psiquiatr Rio Gd Sul. 2004;26:190-4.

34. Mataix D, Lawrence NS, Wooderson S, Speckens A, Phillips ML. The Maudsley obsessive-compulsive stimuli set (MOCCSS): validation of a standardized paradigm for symptom especific provocation in obsessive-compulsive disorder (OCD). Psychiatr Res. 2009;168: 238-41.

35. Fontenelle JM, Santana L, Lessa L, Victoria MS, Mendlowicz MV, Fontenelle LF. O problema do insight em pacientes com transtorno obsessivocompulsivo. Rev Bras Psiquiatr. 2010;32:77-81.

36. Matsunaga H, Kiriike N, Matsui T, Iwasaki Y, Koshimune K, Ohya K, et al. A comparative study of clinical features between pure checkers and pure washers categorized using a lifetime symptom rating method. Psychiatry Res. 2001;105:221-9.

37. Torres AR, Lima MCP. Epidemiologia do transtorno obsessivo-compulsivo. Rev Bras Psiquiatr. 2005;27:237-42. 
38. Torresan RC, Smaira SI, Ramos-Cerqueira ATA, Torres AR. Qualidade de vida no transtorno obsessivo-compulsivo: uma revisão. Rev Psiq Clín. 2008;35:13-9.

39. Mansur CGS, Cabral SB, Sartorelli MCB, Lopes AC, Miguel EC, Bernik MA, et al. Aplicação da estimulação magnética transcraniana de repetição no tratamento do transtorno obsessivo-com- pulsivo e outros transtornos de ansiedade. Rev Psiq Clín. 2004;31 257-61.

40. Chamberlain SR, Blackwell AD, Fineberg NA, Robbins TW, Sahakian BJ The neuropsychology of obsessive compulsive disorder: the importance of failures in cognitive and behavioural inhibition as candidate endophenotypic markers. Neurosci Biobehav Rev. 2005;29:399-419. 